\title{
Open problems: Descending cohomology, geometrically
}

\section{compiled by Barry Mazur*}

Open problems in mathematics, statistics, theoretical computer science and related areas are welcome. Please contact the section editor Kefeng Liu (liu@math.ucla.edu).

The Editors

Open Problem 2014001 (Number theory) Proposed by Barry Mazur, Harvard University, USA.

I was asked by the organizers of the August 2011 birthday conference in honor of Joe Harris to give a short presentation in the session on "Open Problems" in the conference. Now, a great thing when you work together with Joe is that you find yourself in the midst of loads of inspiring problems, thanks to his deep engagement with, and intense curiosity about, all aspects of his subject. He's a master of formulating problems on so many levels that it's already something of an open problem simply to choose just one or two of them in his honor.

Sometimes Joe introduces a problem very broadly and somewhat obliquely, as when he once asked "How many curves are there defined over Q?" Of course, this was an invitation to discuss the dimension-as a function of the genus $g$ - of the Zariski closure of the set of Q-rational points in $M_{g}$ the moduli space of curves of genus $g$. Hyperelliptic curves already gives you $2 / 3$ of the dimension of that moduli space $(\bmod \mathrm{O}(1)$, and as $g \rightarrow \infty)$ but can you get, say, a better fraction than that? This question, of course, immediately connects, via celebrated conjectures of Lang, to questions regarding the algebraic geometric structure of $M_{g}$.

\footnotetext{
* Department of Mathematics, Harvard University

E-mail: mazur@math.harvard.edu
}

And sometimes, when you come to him with a general question-even a vague unformed one-he will clue you in to a rich tradition of very concrete instantiations of it. This happened recently when John Tate, Mark Kisin, and I asked him a certain general question. In the remaining minutes of my presentation today I'll hint at that question and mention briefly at least one specific instance of it that Joe and Mike Roth and Jason Starr had investigated (also Joe's student Arnav Tripathy) opening up, it seems to me, a wide area of excellent algebro-geometric structure.

The general title of the problem, then, could be the title of these notes: descending cohomology, geometrically. Take, for example, a smooth projective threefold ${ }^{1} X$ over $\mathbf{Q}$ with the property that it possesses no holomorphic everywhere regular differential 3-forms; i.e., $h^{0,3}=h^{3,0}=0$. For any prime number $\ell$ let

$$
\left.\mathbf{Q}_{\ell}(1)\right):=\mathbf{Q}_{\ell} \otimes_{\mathbf{z}_{\ell}} T \mu_{\ell^{\infty}},
$$

that is, we've "Tate-twisted" the constant sheaf.

Now let

$$
H_{\ell}:=H_{\ell}(X):=H_{\mathrm{et}}^{3}\left(X \otimes_{\mathbf{Q}} \overline{\mathbf{Q}} ; \mathbf{Q}_{\ell}(1)\right)
$$

denote the three-dimensional $\ell$-adic étale cohomology group of $X$ viewed as $G_{\overline{\mathbf{Q}}}:=\mathrm{Gal}(\overline{\mathbf{Q}} / \mathbf{Q})$-representation, but "descended" in the sense that it is twisted as indicated.

The reason why it is reasonable to do this is that for all primes $p$ of good reduction for $X$, the eigen-

\footnotetext{
${ }^{1}$ We're fixing dimension three and base field $\mathbf{Q}$ just for specificity since we have an interesting open problem even in this level of generality, but-of course-one could consider more general contexts such as odd-dimensional varieties of "niveau de Hodge un," as defined by Deligne in [7].
} 
values of Frobenius in the original untwisted representation are algebraic integers divisible by $p$ (since $h^{0,3}=0$ : this is a consequence, for example, of a conjecture of Katz that is now a theorem; see specifically [4]; for explicit results regarding divisibility, see [6]; for related issues see also [15], [16]) and therefore the downward twisted representation has the property that these eigenvalues divided by $p$ remain algebraic integers, and therefore-in the usual sense-are "Weil numbers" of absolute value $p / 2$.

Even better, the $\ell$-adic Hodge numbers of the downward twisted representation are zero except for $h^{0,1}=h^{1,0}$. That is, given these numerical invariants, the downward twisted representation could conceivably be the Galois representation attached to an isogeny class of an abelian variety over $\mathbf{Q}$. By Faltings' Theorem, if there is such an isogeny class it is unique (also independent of $\ell$ ). Call "it" (whether it exists or not) the phantom isogeny class of abelian varieties over $\mathbf{Q}$ related to this $X$. Within this isogeny class (over $\mathbf{Q}$ ) - if it exists!-there is a unique isomorphism class of abelian varieties, $J(X)_{\mathbf{Q}}$, possessing $\operatorname{Gal}(\overline{\mathbf{Q}} / \mathbf{Q})$-equivarient isomorphisms:

$$
H_{\ell}=H_{\mathrm{et}}^{3}\left(X_{\overline{\mathbf{Q}}} ; \mathbf{Z}_{\ell}(1)\right) \simeq H^{1}\left(J(X)_{\overline{\mathbf{Q}}} ; \mathbf{Z}_{\ell}\right)
$$

for all primes $\ell$. Here the subscript $\bar{Q}$ refers to base change from $\mathbf{Q}$ to $\overline{\mathbf{Q}}$, and the Galois action is the natural one.

It is known that much of this phantom abelian variety actually does exist. For example:

- The complex abelian variety: The intermediate jacobian construction (cf. [5] ) gives us a complex torus,

$$
\begin{aligned}
J\left(X_{\mathbf{C}}\right): & =H^{3}\left(X_{\mathbf{C}}, \mathbf{Z}\right) \backslash H^{3}\left(X_{\mathbf{C}}, \mathbf{C}\right) / H^{2,1}\left(X_{\mathbf{C}}\right) \\
& =H^{2,1}\left(X_{\mathbf{C}}\right) / H^{3}\left(X_{\mathbf{C}}, \mathbf{Z}\right),
\end{aligned}
$$

and cup-product provides a Riemann form on $J(X)_{\mathbf{C}}$ rendering it a principally polarized abelian variety over $\mathbf{C}$ that is (the conjectural candidate for) the complex analytic abelian variety associated to the putative phantom. (Also, if $X_{\mathbf{C}}$ varies algebraically over a parameter space-over $\mathbf{C}-$ so does this principally polarized abelian variety $J(X)_{\mathbf{C}}$.)

- The abelian variety over $\mathbf{F}_{p}$ : For every prime $p$ of good reduction, there really does exist a unique isogeny class, $\mathcal{J}(X)_{\mathbf{F}_{p}}$, of abelian varieties over $\mathbf{F}_{p}$ that would play the role of the reduction $\bmod p$ of the phantom if the latter were to exist. ${ }^{2}$ This

2 It might be fun to look for a somewhat direct construction of an abelian scheme $J(X)_{\mathbf{z}_{p}}$ over Spec $\mathbf{Z}_{p}$ whose special fiber is in the isogeny class $\mathcal{J}(X)_{\mathbf{F}_{p}}$ and for which the $p$-adic $\operatorname{Gal}\left(\overline{\mathbf{Q}}_{p} / \mathbf{Q}_{p}\right)$ representation on $H_{\mathrm{et}}^{1}\left(J(X)_{\mathbf{Q}_{p}} ; \mathbf{Q}_{p}\right)$ is-when appropriately twisted-isomorphic to the representation of $\operatorname{Gal}\left(\overline{\mathbf{Q}}_{p} / \mathbf{Q}_{p}\right)$ on $H_{\mathrm{et}}^{3}\left(X_{\overline{\mathbf{Q}}_{p}} ; \mathbf{Q}_{p}\right)$. follows from our discussion above, plus the theorems of Tate and $\operatorname{Honda}^{3}$ ([17], [18], [9]) that guarantee that conjugacy classes of Weil numbers of absolute value $p / 2$ correspond bijectively to isogeny classes of simple abelian varieties over $\mathbf{F}_{p}$.

- Descent of the complex abelian variety by the method of "irreducibility of the monodromy action": If $X$ is a complete intersection with the above properties (i.e., $h^{0,3}(X)=h^{3,0}(X)=0$ ) then Deligne ([7]) established the existence of a unique (principally polarized) abelian variety $J(X)_{\mathbf{Q}}$ of the sort we seek. The method is quite conceptual, and depends on irreducibility of monodromy actions for the universal family of which $X$ is a member. It works - mutatis mutandis-for complete intersections $X$ of any (odd) dimension $n=2 m+1$ such that $X$ is of "level one" in the sense that its only nonzero $n$-dimensional Hodge numbers are the middle two: $h^{m, m+1}$ and $h^{m+1, m}$. More specifically, let $S$ be the universal family of smooth complete intersections of dimension $n$ cut out in $\mathbf{P}^{n+v}$ by $v$ homogeneous forms of degrees $a_{1}, a_{2}, \ldots, a_{v}$ and assume that these smooth complete intersections are of "level one" and that $X$ is a member of that family corresponding to the point $s \in S$. As mentioned above, the major property that Deligne relies on for the construction of $J(X)_{\mathbf{Q}}$ is that the monodromy actions of $\pi_{1}(S, s)$ on $H^{3}\left(X_{\mathbf{C}} ; \mathbf{Q}\right)$ and on $H^{3}\left(X_{\mathbf{C}} ; \mathbf{Z} / \ell \mathbf{Z}\right)$ (for every prime number $\ell$ ) are all absolutely irreducible.

There has been some beautiful recent work developing the theme that absolutely irreducible monodromy sometimes allows one to descend fields of definition from $\mathbf{C}$ to number fields while keeping control of the natural Galois actions, thereby constructing natural arithmetic models of polarized abelian varieties associated to Hodge structures. Starting with period maps ${ }^{4}$ related to Hodge structures for various moduli problems-as developed in recent work of AllcockCarlson-Toledo ([1], [2]), Kondo ([10], [11]), and Looijenga-Swierstra ([14]) Kudla and Rapoport ([13]) descend fields of definition (via the technique irreducibility of monodromy) in a number of cases.

\footnotetext{
${ }^{3}$ For another very nice exposition of this, see also Theorem 4.1(1) of Kirsten Eisenträger's exposition article "The theorem of Honda and Tate" which can be found on her web page. 4 These are called occult period maps since they aren't always the straightforward evident map associating to the algebraic variety its natural Hodge structure; at times-for example-one rather makes an intermediate construction such as viewing the variety of interest as the branch locus of a cyclic cover of the ambient projective space, and then passing to the Hodge structure of that cyclic cover.
} 
- Construction of the phantom abelian variety by direct algebro-geometric means:

1. Prym constructions: For a smooth cubic threefold $X$, the classical construction due to Mumford gives its intermediate jacobian as the Prym abelian variety of a certain curve constructed from the cubic threefold. Roughy, one blows $X$ up at a line $L \subset X$ and exhibits this blow-up as a conic bundle over the complement of a curve $C$ projective space; the curve $C$ has a double cover $\tilde{C}$ (see Jeff Achter's article [3]) that establishes a Prym construction-extending the classical work of the intermediate Jacobian of a cubic threefold over an algebraically closed field.

2. Intermediate Jacobian as Albanese: The classic paper of Clemens and Griffiths [5] established that the intermediate jacobian of a smooth cubic threefold $X$ in $P^{4}$ is the Albanese variety of the Fano scheme of lines on $X$.

With this latter route of construction as a possible proto-type we were led to ask Joe:

\begin{abstract}
Question 1. Can the intermediate jacobian, i.e., this phantom abelian variety, be constructed as-or at least in terms of-the Albanese variety of some Hilbert scheme geometrically attached to $X$ ?
\end{abstract}

When we asked Joe about this, he responded:

Mike Roth, Jason Starr and I looked at an example of this, the cubic threefold $X$ in $\mathbf{P}^{4}$ (Abel-Jacobi maps associated to smooth cubic threefolds): in this case, it's known that the Albanese variety of the Fano scheme of lines on $X$ is what you want; we wanted to see if the same was true for the space of rational curves of any degree. We were able to verify this up to degree 5 , but not in general.

Part of the motivation, I should add, was the possibility of applying a similar construction to something like the cubic fourfold, where we don't have any sort of geometric object associated to the Hodge structure.

A natural place to look for these intermediate jacobians, at least for threefolds $X$ that are uniruled ${ }^{5}$ is as Albanese varieties attached to, say, appropriate Kontsevich spaces of parametrized genus zero stable curves in $X$; or Albanese varieties attached to variants of Kontsevich spaces, such as in the work of Harris Roth, and Starr quoted above, where one takes Albanese varieties of Hilbert schemes classifying curves

\footnotetext{
${ }^{5}$ Uniruled means that for every point $x$ of $X$ there exists a rational curve lying in $X$ passing through $x$. The condition $h^{3,0}=0$ for a threefold is not enough to guarantee that it is uniruled; there is a conjecture that if no positive power of the canonical bundle of $X$ has sections, then $X$ is uniruled. This is conjectured in all dimensions, and true for curves and surfaces.
}

of genus zero (with specific attributes) in $X$. Given that there are variant possibilities, one is led to ask questions of stability, ${ }^{6}$ such as:

Question 2. Consider the Kontsevich space ${ }^{7} \mathcal{K}(X, \beta)$ where $X$ is a smooth three-dimensional hypersurface and $\beta \in H_{2}(X, \mathbf{Z})$ a chosen homology class. Here $\beta$ is the fundamental class of the rational curves in $X$ that $\mathcal{K}$ parametrizes; in the case of smooth hypersurfaces, then, $\beta$ is simply the degree of these rational curves. Let $J(X ; \beta):=$ the Albanese variety of the Kontsevich space $\mathcal{K}(X, \beta)$. How does $J(X ; \beta)$ depend on $\beta$ ?

\section{References}

[1] D. Allcock, J. A. Carlson and D. Toledo, The complex hyperbolic geometry of the moduli space of cubic surfaces, J. Algebraic Geom. 11 no. 4, 659-724, (2002).

[2] D. Allcock, J. A. Carlson and D. Toledo, The moduli space of cubic threefolds as a ball quotient, arXiv:math/0608287.

[3] J. Achter, Arithmetic Torelli maps for cubic surfaces and threefolds, arXiv:1005.2131v3 [math.AG] 29 Feb 2012.

[4] P. Berthelot and A. Ogus, Notes on crystalline cohomology. Mathematical Notes 21 Princeton University Press, Princeton, N.J.; University of Tokyo Press, Tokyo (1978).

[5] C. H. Clemens and P. A. Griffiths, The intermediate Jacobian of the cubic threefold, Annals of Mathematics Second Series 95 (2) 281-356, (1972).

[6] P. Berthelot, H. Esnault and K. Rülling, Rational points over finite fields of regular models of algebraic varieties of Hodge Type $\geq 1$, arXiv:1009.0178v2, Sept 2011.

[7] P. Deligne, Les intersections complètes de niveau de Hodge un, Invent. Math. 15, 1972. 237-250.

[8] W. Fulton and R. Pandharipande, Notes on stable maps and quantum cohomology. In Algebraic geometry Santa Cruz 1995, 62 Part 2 of Proc. Sympos. Pure Math., pp. 45-96. Amer. Math. Soc. (1997).

[9] T. Honda, Isogeny classes of abelian varieties over finite fields. J. Math. Soc. Japan 20, 83-95 (1968).

[10] S. Kondo, A complex hyperbolic structure for the moduli space of curves of genus three, J. Reine Angew. Math. 525, 219-232 (2000).

[11] S. Kondo, The moduli space of curves of genus 4 and Deligne-Mostow's complex reflection groups, Algebraic geometry 2000, Azumino (Hotaka), pp. 383-400, Adv. Stud. Pure Math. 36, Math. Soc. Japan Tokyo (2002).

[12] M. Kontsevich, Enumeration of rational curves via torus actions, pp. 335-368 in The moduli space of curves Progr. Math., 129, Birkhäuser, Boston, MA (1995).

[13] S. Kudla and M. Rapoport, On occult period maps, arXiv:1203.1272v1 [math.AG] 6 Mar 2012.

[14] E. Looijenga and R. Swierstra, The period map for cubic threefolds, Compos. Math. 143, no. 4, 1037-1049 (2007).

\footnotetext{
${ }^{6}$ As Joe explained to me, the corresponding "stability question" for fourfolds would involve the MRC quotients of the corresponding Kontsevich spaces and would have a very different look.

7 In a more general context, for $g \geq 0$ and $k$ a non-negative integer $\geq 3-g$, and for $X$ an algebraic variety over $\mathbf{C}$ with $\beta \in H_{2}(X, \mathbf{Z})$ the Kontsevich space $\bar{M}_{g, k}(X, \beta)$ as in [12] is defined to be the moduli space that classifies stable curves of genus $g$ with $k$ marked points and a map to $X$ that sends its fundamental class to $\beta$. Here we are denoting $\bar{M}_{g, k}(X, \beta)$ simply $\mathcal{K}(X, \beta)$.
} 
[15] B. Mazur, Frobenius and the Hodge filtration, Bull. Amer. Math. Soc. 78, 653-667 (1972).

[16] A. Ogus, Frobenius and the Hodge spectral sequence, Advances in Mathematics 162, 141-172 (2001).
[17] J. Tate, Endomorphisms of abelian varieties over finite fields. Invent. Math. 2, 134-144 (1966).

[18] J. Tate, Classes d'isogénie des variétés abéliennes sur un corps fini. Séminaire Bourbaki 21, 95-110 (1968). 